\title{
Nanofibers and their biomedical use
}

\author{
ROMANA ROŠIC \\ PETRA KOCBEK \\ JAN PELIPENKO \\ JULIJANA KRISTL \\ SAŠA BAUMGARTNER*
}

University of Ljubljana, Faculty of Pharmacy, Department of Pharmaceutical Technology, 1000 Ljubljana, Slovenia
The idea of creating replacement for damaged or diseased tissue, which will mimic the physiological conditions and simultaneously promote regeneration by patients' own cells, has been a major challenge in the biomedicine for more than a decade. Therefore, nanofibers are a promising solution to address these challenges. These are solid polymer fibers with nanosized diameter, which show improved properties compared to the materials of larger dimensions or forms and therefore cause different biological responses. On the nanometric level, nanofibers provide a biomimetic environment, on the micrometric scale three-dimensional architecture with the desired surface properties regarding the intended application within the body, while on the macrometric scale mechanical strength and physiological acceptability. In the review, the development of nanofibers as tissue scaffolds, modern wound dressings for chronic wound therapy and drug delivery systems is highlighted. Research substantiates the effectiveness of nanofibers for enhanced tissue regeneration, but ascertains that evidences from clinical studies are currently lacking. Nevertheless, due to the development of nano- and bio-sciences, products on the market can be expected in the near future.

Keywords: nanofibers, tissue engineering, wound dressings, drug delivery systems, analogues of ECM

Giant progress of nanoscience in the last decade has prompted the development of new nanosystems as well as new technologies for their preparation. Polymer nanofibers prepared by the electrospinning method are just one of such examples. These are solid fibers with diameters from a few tens up to $1000 \mathrm{~nm}$ (Fig. 1) and theoretically unlimited length having a huge surface area per unit mass and small pore size. All these parameters strongly depend on the preparation process and hence can vary significantly. Pore size for example is the crucial parameter when using nanofibers as tissue scaffolds or wound dressings. Namely, when pores are smaller than the cell size, the cells are cap-

\footnotetext{
* Correspondence; e-mail: sasa.baumgartner@ffa.uni-lj.si
} 
tured and their spreading across and through nanofibers is limited. Additionally, nanofibers express greater flexibility and mechanical strength compared to the same material of other forms and larger dimensions $(1,2)$.

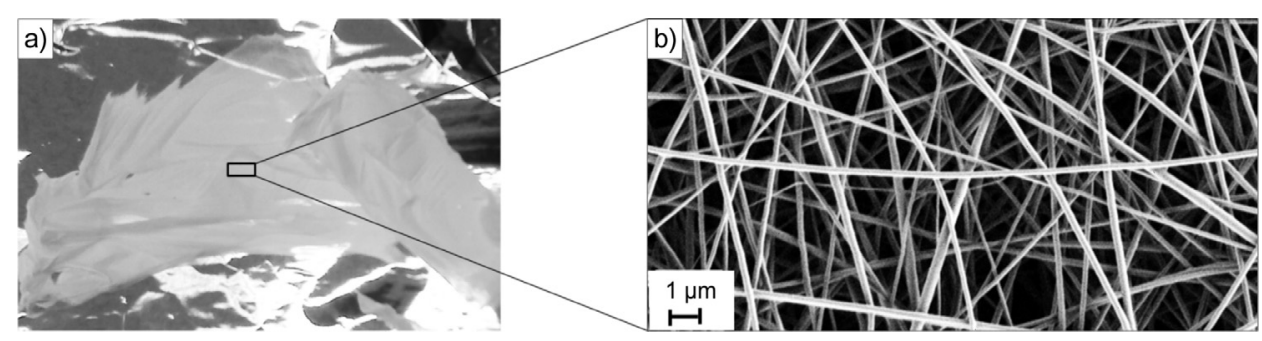

Fig. 1. View of polymer nanofibers collected on an aluminum collector with: a) the naked eye and b) magnified picture of the same sample under scanning electron microscope.

A bibliometric analysis in the Web of Science database picturesquely reveals the significance of the interest in polymer nanofibers as the number of scientific publications raises from only about a hundred at the beginning of the decade to more than 2500 in 2011 (Fig. 2) (3). The importance of nanofibers is further supported by the fact that the global market for nanofibers in all industrial areas is estimated to have been worth 176 million dollars in the year 2012. The annual growth of the market is even expected to be $30 \%$ despite the fact that there are only about 50 companies in the world involved in the production of nanofibers (4).

Unique properties of nanofibers resulting from their structure coupled with the relative ease of their preparation technology extended the applicability of nanofibers in various fields, but with the strongest emphasis on the development of nanofibers for medical purposes, air filtration and electronics $(1,5)$.

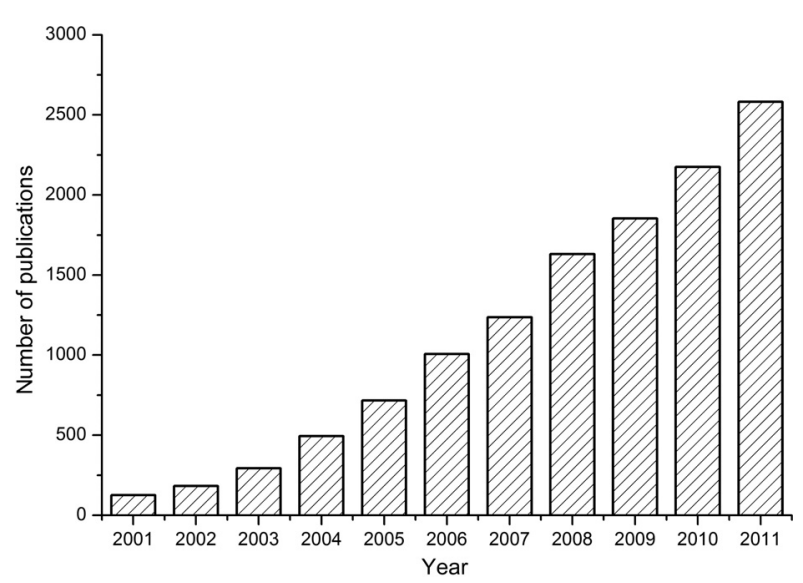

Fig. 2. Increase in the number of scientific publications from the nanofiber field during the period 2001-2011 (3). 
Based on a literature survey, previous research in the biomedical field, which is the subject of this review, can be classified into three groups, namely, the development and usage of nanofibers as i) tissue scaffolds, ii) modern wound dressings and iii) drug delivery systems (5-8). Without any doubt nanofibers are a unique class of materials, since on the nanometric level they provide a biomimetic environment, on the micrometric level three-dimensional architecture with the desired surface properties regarding the intended application within the body, while on the macrometric scale mechanical strength and physiological acceptability. Nanofibers can be prepared from polymers of both synthetic and natural origin, but among them those being biocompatible and biodegradable are preferred $(2,9)$. Although various methods, such as self-assembly, phase separation or electrospinning, can be used for their preparation, only the electrospinning technology, which uses high voltage supply to transform a polymer solution into solid nanofibers, is versatile, efficient, widely used and applicative on the industrial scale (Fig. 3) $(10,11)$.

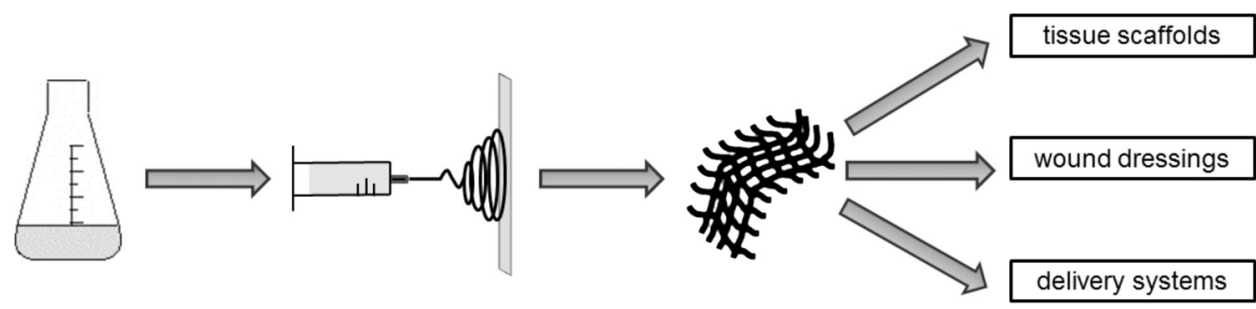

Fig. 3. Scheme of nanofiber preparation from polymer solution by electrospinning and their potential use in biomedicine.

\section{Nanofibers in regenerative medicine}

Tissue engineering, also named regenerative medicine, is a promising multidisciplinary science field. Its main objective is the development and construction of a biocompatible tissue scaffold, which will, in combination with living cells and/or bioactive substances, renew, replace, maintain or heal damaged cells or tissues and stimulate the physiological process of regeneration (12). Despite the fact that tissue engineering has been constantly evolving ever since the first mention of the term in 1987, the clinical use of tissue substitutes is nowadays still far below expectations (13). The primary idea of regenerative medicine was to prepare a carrier from biomaterials in the form of the affected tissue or organ, seed it with the patient's autologous cells and then implant the entire system in the body (14). However, another strategy is currently in precedence. This is the application of a porous three-dimensional substitute that has the ability to mimic the natural extracellular matrix (ECM) by itself (12). The underlying rationale is that different injuries or pathological conditions cause a change in the ECM in the body and cells have to restore it during the regeneration process. Unfortunately, physiological 
ECM formation is often insufficient or even disrupted and it is therefore reasonable to expect that the application of an ECM analogue will enhance natural tissue repair, since the tissue will adopt the applied scaffold as its own $\operatorname{ECM}(5,15)$.

Nonetheless, the preparation of an efficient ECM analogue is a tough challenge. A competent analogue must not only imitate the topography and structural properties of the natural ECM, but it should also have the ability to connect with the cells in all three dimensions and promote communication between them (16). The physical structure of the natural ECM is in nanometer dimensions and consists of three basic groups of macromolecules. Namely, filamentary structural proteins such as collagen and elastin fibers (50-500 nm), which provide the structure and strength of the matrix; adhesive glycoproteins, which connect the matrix parts with each other and with the cells; and proteoglycans and hyaluronan, which provide flexibility and lubrication of the matrix (5). ECM is the essential and most extensive component of every tissue because it surrounds the cells and is produced by tissue cells themselves. It is much more than just a physical support for the cells. ECM also represents a substrate for cell adhesion via specific ligands, provides and directs cell migration and regulates their growth and functions through a variety of bioactive factors $(14,15)$.

Currently, the most promising analogues of ECM are nanofibers, since their nanoscale fibrous structure mimics the fibrillar elements of ECM in so far the most genuine way (17). Even more, detailed investigation reveals that the majority of human tissues and organs on the nanometric level are actually hierarchically organized fibrous structures $(18,19)$. Thus, after implantation the nanofibers unite with the surrounding tissue, provide efficient adhesion of the cells as well as their proliferation, differentiation and migration, support neovascularization and, owing to their porosity, enable the exchange of nutrients and metabolites $(1,5,20)$.

The biomimetic effect of nanofibers can be further promoted by a proper selection of the polymer from which they are prepared. For this purpose, polycaprolactone, poly(lactic acid), poly(glycolic acid) and their copolymers as well as collagen, hyaluronic acid, natural polymers of ECM and many others have been studied (13). Besides, optimization of fibers' thickness, alignment and porosity enable the formation of a microstructure that completely fits into a specific place in the body. For example, studies have shown that a thick layer of nanofibers should be used for a good response to the pulsating flow in the artery wall $(21,22)$, while real conditions in the vessels and nerves are better imitated with structurally oriented, rather than randomly deposited, nanofibers (Fig. 4) $(18,22)$. In particular, the arrangement and orientation of nanofibers considerably affect the cell response, since studies have proven that cells grown on aligned fibers orient in the direction of the fibers and frequently also reflect a higher proliferation rate $(22,24)$.

Surface functionalization is an additional way of upgrading nanofibers; it can be easily achieved by embedment or adsorption of components, such as growth factors and glycosaminoglycans (hyaluronic acid, dermatan sulphate, chondroitin sulphate, heparin, etc.). In particular, incorporation of the latter is an important aspect in the pristine imitation of ECM, since glycosaminoglycans have many crucial features, including linking of collagen fibers and binding of different growth factors (26). Interestingly, beside these components, live cells have also been incorporated into thicker fibers (27). 

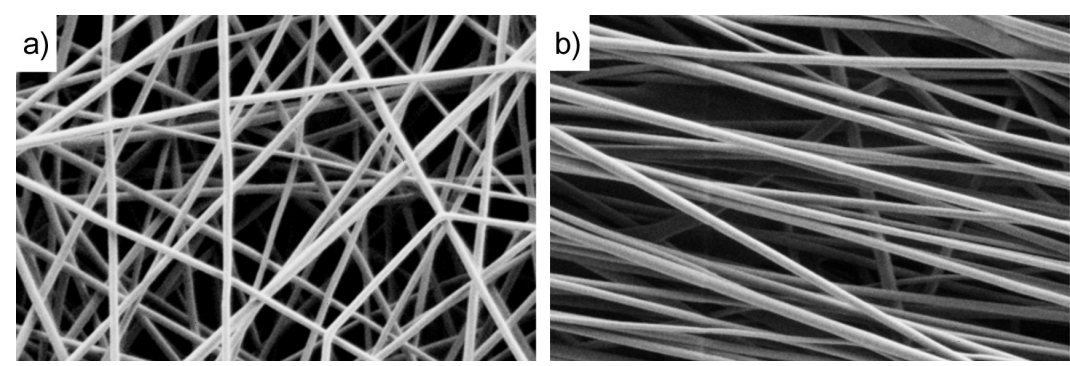

Fig. 4. Representative figure of: a) randomly collected and b) aligned nanofibers with parallel orientation under scanning electron microscope.

Several independent studies on cell cultures or on experimental animals have already proven that nanofibers represent a forward-looking approach for the engineering of various tissues, such as bone, blood vessels, nerves and others (1). Nevertheless, despite numerous scientific publications in this area, clinical studies confirming their effectiveness in humans are not yet available.

\section{Nanofibers as advanced bone substitutes}

As damaged bones and osteoporotic fractures are a serious health problem, a significant number of researchers focus on the development of nanofibers as bone substitutes (5). Most drugs used to treat osteoporosis today, such as bisphosphonates, estrogen, raloxifene and calcitonin, only slow the degradation of the bone, while the formation of a new one is not encouraged (28). Notably, the latter can be significantly influenced by the application of a substitute made of nanofibers $(28,29)$. Human bones are made up of $35 \%$ organic matter, mainly collagen type I, and $65 \%$ inorganic substances, where calcium phosphate predominates. In view of this, beside the polymer forming the fibrous frame, advanced bone substitutes include hydroxyapatite, which strengthens the structural integrity of the substitute and enables good osteoconductivity and binding of the natural bone components on the substitute (30). In vitro studies have confirmed a high degree of osteoblast attachment and growth on nanofibers from chitosan and hydroxyapatite as well as a significantly increased mineralization as soon as in 15 days (31).

Further, different studies have demonstrated the benefit of nanofibers for reparation of damaged ties between the bones due to their large surface area and porosity (5). Investigations have also compared different systems of the same material for this purpose and have proven that better attachment and proliferation of human ligament fibroblasts is achieved on the polyurethane nanofibers than on film and that a progressive collagen synthesis in vitro is better stimulated when using oriented nanofibers than those randomly deposited (31).

To conclude, nanofibrous bone substitutes currently only improve the quality of bone integrity due their structure, but the development trend suggests that the next generation of substitutes will include active substances that will additionally enhance bone regeneration. 


\section{Nanofibers as progressive dressings for chronic wound therapy}

One to two percentages of the total population in the industrialized world experience a chronic wound at least once in a lifetime. Although a number of novel treatments, such as therapy with hyperbaric oxygen and negative pressure, did improve the results of their treatments, an extensive and chronic wound still remains a major complication and cause of death in diabetic and hospitalized patients as well as by those having large burns $(18,24,32,33)$.

Chronic wounds are defined as all treated wounds that do not heal during an eight-week period because their natural healing process is delayed or disrupted. The latter is most commonly stuck in the inflammatory phase, when secretion of cytokines and growth factors as well as extensive proteolysis and unsatisfactory neovascularization are present in the wound (35). The main reason why a chronic wound is not able to close is the imbalance between the tissue construction and degradation processes at the wound site and especially inadequate formation of a functional ECM, which otherwise encourages, directs, and organizes the healing. Therefore, the development of modern wound dressings, which mimic exactly the missing matrix, is the most comprehensible approach for the treatment of non-healing wounds. For the aforementioned reasons, nanofibers can be used as efficient ECM analogues. After the application onto the wound, cells will take the latter as if synthesized by themselves and consequently oriented chemotaxis, adhesion, differentiation and cell growth will be stimulated as well as the formation and deposition of a provisional matrix and re-epithelialization $(16,18,36)$.

Inclusion of nanofibers as components of a modern wound dressings combines a number of advantages. They replace elastin and collagen fibers of ECM in terms of shape, size and mechanical properties, permit drainage of wound exudates, are gas permeable and prevent wound contamination. Results have shown that the nanofiber topography itself, regardless of the material nanofibers are made of, has a positive impact on cell behaviour since it stimulates cellular regeneration processes (13). Nevertheless, if nanofibers are made of hydrophilic materials, they have an added value of providing a moist wound environment, which is proven to fasten the migration of epithelial cells from the wound edge toward the center and ameliorate healing (36).

One of the recent studies by Balaji et al. has shown that application of nanofibers made of proteolysis-resistant angiogenic RAD16-II peptide in diabetic ulcers results in in situ formation of a provisional matrix composed of nanofibers and ECM elements as soon as three days after the wound occurrence, which consecutively leads to considerably increased wound closure by endothelial cells and formation of granulation tissue. After 28 days, the thickness of the reconstituted epidermis is similar to that of the native skin; there are evidences of hair follicle and absence of hypertrophic scar formation (37).

Of particular interest are also nanofibers made of hyaluronic acid (HA), because concentration of the latter in the wound increases significantly during the early stages of the healing process. HA accelerates cell proliferation, induces migration of fibroblasts and cells of the immune system at the wound site, moderates the inflammatory process, reduces the scarring intensity and even has antioxidative properties (38). All these effects are primarily attributable to the physical and chemical properties of HA, especially its exceptional hydrophilicity, and partly also to the binding on specific receptors located on the cells and ECM. Comparative research based on histopathological tests has proven 
that wound healing with HA nanofibers is better than with gauze, HA alone or adhesive dressings, while compared to the antibiotic treatment, no statistically significant differences were observed (38). In addition, other studies taking into consideration nanofibers from different polymers and established materials for wound dressings, such as Tega$\operatorname{derm}^{\circledR}$, a nylon dressing, and hydrogels, have led to the same conclusion - nanofibers make the most effective and advanced wound dressings (36).

To even more actively participate in the regeneration process and further enhance healing, different active substances can be included inside or on the surface of nanofibers (39). An additional benefit is that with such dressing a wound is treated locally with direct contact between the drug and tissue, with limited possibility of systemic side effects (40).

Active substances can be incorporated inside the nanofibers, physically adsorbed or chemically bound to the surface. Of the various loading possibilities, physical entrapment is currently the most widespread, since the drug in the nanofibers is protected against unfavorable environmental conditions and it offers good control over the drug release (8). Moreover, incorporation of a drug in nanofibers is relatively easy to perform, since the drug is simply dissolved in the polymer solution prior to electrospinning and the formation of an amorphous drug is favoured due to a very limited time being available for drug recrystallization during the fiber formation $(9,11)$. A typical release profile from such nanofibers exhibits an initial burst effect followed by an almost linear, sustained release $(1,5)$. In addition, core-shell nanofibers can be prepared, which provides a drug-reservoir system with a shell barrier protecting the incorporated drug and controlling the drug diffusion rate.

The first drug that was incorporated into nanofibers from poly(lactic acid) and a copolymer of polyethylene and vinyl acetate was an antibiotic tetracycline (41). Since then, many other antibiotics $(40,42)$ as well as various antiseptics (43), antifungals (5), non-steroidal anti-inflammatory agents $(44)$, anti-cancer drugs $(40,45)$ as well as biomolecules such as proteins $(5,7,21,46)$ and nucleic acids (47) have been built into or adsorbed on nanofibers. For example, Choi and his colleagues bound the recombinant human epidermal growth factor on the surface of polycaprolactone and poly(ethylene oxide) nanofibers and proved that the growth factor retained its biological activity (7). In addition, using an animal model of diabetic ulcers they found out that such dressing stimulated the proliferation and differentiation of keratinocytes at the early stages of wound healing, while their phenotype remained unchanged. The in vitro investigation of Yang et al. indicated that nanofibers loaded with fibroblast growth factor enhanced adhesion, proliferation and secretion of ECM of mouse embryonic fibroblasts. After an initial burst effect, a gradual release over about four weeks was achieved, whereas re-epithelialization and mature capillary vessels were generated after 14 days (46). The latest approach in managing bacterial infections in chronic wounds is the embedment of silver nanoparticles as effective antimicrobial agents into nanofibers (48).

\section{CONCLUSIONS}

Examples described in this review are only a minor proportion of all the results proving the great potential and usefulness of nanofibers. On the other side, construction of a three-dimensional tissue scaffold with suitable thickness, strength and mesh size for 
adequate cell infiltration, which is essential for efficient in vivo application, still remains an impressive challenge. Nevertheless, nanofibers with their physicochemical properties and nanotopography display improvements in the fields of tissue engineering, wound therapy and drug delivery systems. According to the emerging trend, where new discoveries and patents daily promote new knowledge about nanofibers' ability of faster and more complete tissue repair, clinical studies are soon to be expected. Consecutively, the development is anticipated to be directed towards the selection of specific polymer elements, which will further ameliorate the biomimicry for EMC, as well as to the functionalization of nanofibers for particular purposes and thereby expansion of their application areas.

Acknowledgements. - The authors are grateful to the Slovenian Research Agency for financial support of their research work: P1-634 0189, J1-4236, 1000-09-310085 and 1000-11-310213.

\section{REFERENCES}

1. S. Ramakrishna, K. Fujihara, W. E. Teo, T. C. Lim and Z. Ma, An introduction to electrospinning and nanofibers, World Scientific, New Jersey 2005.

2. R. Rošic, P. Kocbek, S. Baumgartner and J. Kristl, Electrospun hydroxyethylcellulose nanofibers: the relationship between structure and process, J. Drug Del. Sci. Tech. 21 (2011) 229-236.

3. ISI Web of Knowledge, http://apps.isiknowledge.com; access date September 3, 2012.

4. BCC Research 2010 Report Nano43B.

5. T. J. Sill and H. A. von Recum, Electrospinning: applications in drug delivery and tissue engineering, Biomaterials 29 (2008) 1989-2006; DOI: 10.1016/j.biomaterials.2008.01.011.

6. B. S. Kim, I. K. Park, T. Hoshiba, H. L. Jiang, Y. J. Choi, T. Akaike and C. S. Cho, Design of artificial extracellular matrices for tissue engineering, Prog. Polym. Sci. 36 (2010) 238-268; DOI: 10. 1016/j.progpolymsci.2010.10.001.

7. J. S. Choi, K. W. Leong and H. S. Yaoo, In vivo wound healing of diabetic ulcers using electrospun nanofibers immobilized with human epidermal growth factor (EGF), Biomaterials 29 (2008) 587-596; DOI: 10.1016/j.biomaterials.2007.10.012.

8. P. C. Caracciolo, P. C. Tornello, F. M. Ballarin and G. A. Abraham, Development of electrospun nanofibers for biomedical applications: state of the art in Latin America, J. Biomater. Tissue Eng. 3 (2013) 39-60; DOI: 10.1166/jbt.2013.1066.

9. R. Rošic, J. Pelipenko, J. Kristl, P. Kocbek and S. Baumgartner, Properties, engineering and applications of polymeric nanofibers: current research and future advances, Chem. Biochem. Eng. Q. 26 (2012) 417-425.

10. R. Rošic, J. Pelipenko, S. Baumgartner, M. Bešter-Rogač and J. Kristl, The role of rheology of polymer solutions in predicting nanofiber formation by electrospinning, Eur. Polym. J. 48 (2012) 1374-1384; DOI: 10.1016/j.eurpolymj.2012.05.001.

11. J. Pelipenko, J. Kristl, R. Rošic, S. Baumgartner and P. Kocbek, Interfacial rheology: an overview of measuring techniques and its role in dispersions and electrospinning, Acta Pharm. 62 (2012) 123-140; DOI: 10.2478/v10007-012-0018-x.

12. Y. Tabata, Biomaterial technology for tissue engineering applications, J. R. Soc. Interface 6 (2009) 311-324; DOI: 10.1098/rsif.2008.0448.focus.

13. R. Sridhar, J. R. Venugopal, S. Sundarrajan, R. Ravichandran, B. Ramalingam and S. Ramakrishna, Electrospun nanofibers for pharmaceutical and medical applications, J. Drug Del. Sci. Tech. 21 (2011) 451-468. 
14. W. Ji, Y. Sun, F. Yang, J. J. van den Beucken, M. Fan, Z. Chen Z and J. A. Jansen, Bioactive electrospun scaffolds delivering growth factors and genes for tissue engineering applications, Pharm. Res. 28 (2011) 1259-1272; DOI: 10.1007/s11095-010-0320-6.

15. C. M. Kelleher and J. P. Vacanti, Engineering extracellular matrix through nanotechnology, J. R. Soc. Interface 7 (2010) 717-729; DOI: 10.1098/rsif.2010.0345.focus.

16. C. P. Barnes, S. A. Sell, E. D. Boland, D. G. Simpson and G. L. Bowling, Nanofibers technology: Designing the next generation of tissue engineering scaffolds, Adv. Drug Deliv. Rev. 59 (2007) 1413-1433; DOI: 10.1016/j.addr.2007.04.022.

17. R. Rošic, P. Kocbek, S. Baumgartner and J. Kristl, Electrospun chitosan/PEO nanofibers and their relevance in biomedical application, IFMBE proceedings 37 (2011) 1296-1299; DOI: 10.1007/ 978-3-642-23508-5_335.

18. J. Gunn and M. Zhang, Polyblend nanofibers for biomedical applications: perspectives and challenges, Trends Biotechnol. 28 (2010) 189-197; DOI: 10.1016/j.tibtech. 2009.12.006.

19. V. Beachley and X. Wen, Polymer nanofibrous structures: fabrication, biofunctionalization, and cellinteractions, Prog. Polym. Sci. 35 (2010) 868-892; DOI: 10.1016/j.progpolymsci. 2010. 03.003.

20. G. Zanatta, M. Rudisile, M. Camassola, J. Wendorff, N. Nardi, C. Gottfried and P. Pranke, C. A. Netto, Mesenchymal stem cell adherence on poly(D,L-lactide-co-glycolide) nanofibers scaffold is integrin beta 1 receptor dependent, J. Biomed. Nanotechnol. 8 (2012) 211-218; DOI: 10.1166/jbn. 2012.1382.

21. M. Spasova, O. Stoilova, N. Manolova, I. Rashkov and G. Altankov, Preparation of PLLA/PEG nanofibers by electrospinning and potential applications, J. Bioact. Compat. Polym. 22 (2007) 6276; DOI: $10.1177 / 0883911506073570$.

22. S. G. Kumbar, S. P. Nukavarapu, R. James, M. V. Hogan and C. T. Laurenchin, Recent Patents on Electrospun Biomedical Nanostructures: An Overview, Recent Pat. Biomed. Eng. 1 (2008) 68-78.

23. J. Pelipenko, R. Rošic, P. Kocbek, S. Baumgartner and J. Kristl, The topography of electrospun nanofibers and its impact on the growth and mobility of keratinocytes, Eur. J. Pharm. Biopharm. 84 (2013) 401-411; DOI: 10.1016/j.ejpb.2012.09.009.

24. K. T. Shalumon, D. Sathish, S. V. Nair, K. P. Chennazhi, H. Tamura and R. Jayakumar, Fabrication of aligned poly(lactic acid)-chitosan nanofibers by novel parallel blade collector method for skin tissue engineering, J. Biomed. Nanotechnol. 8 (2012) 405-416; DOI: 10.1166/jbn.2012.1395.

25. Z. Q. Feng, H. J. Lu, M. K. Leach, N. P. Huang, Y. C. Wang, C. J. Liu and Z. Z. Gu, The influence of type-I collagen-coated PLLA aligned nanofibers on growth of blood out growth endothelial cells, Biomed. Mater. 5 (2010) 1-8; DOI: 10.1088/1748-6041/5/6/065011.

26. M. J. P. Bigs, R. G. Richards and M. J. Dalby, Nanotopographical modification: a regulator of cellular function through focal adhesions, Nanomedicine 6 (2010) 619-633; DOI: 10.1016/j.nano. 2010.01.009.

27. M. F. Cabolat, C. Tang, S. H. Bernacki, B. Pourdeyhimi and S. Khan, Mammalian cell viability in electrospun composite nanofiber structures, Macromol. Biosci. 11 (2011) 1346-1356; DOI: 10. 1002/mabi.201100108.

28. J. H. Jang, O. Castano and H. W. Kim, Electrospun materials as potential platforms for bone tissue engineering, Adv. Drug Del. Rev. 61 (2011) 1065-1083; DOI: 10.1016/ j.addr.2009.07.008.

29. J. M. Holzwarth and P. X. Ma, Biomimetic nanofibrous scaffolds for bone tissue engineering, Biomaterials 32 (2011) 9622-9629; DOI: 10.1016/j.biomaterials.2011.09.009.

30. G. Wei and P. X. Ma, Nanostructured biomaterials for regeneration, Adv. Funct. Mater. 18 (2009) 3566-3582; DOI: 10.1002/adfm.200800662.

31. J. R. Venugopal, V. R. GiriDev, T. Senthilram, D. Sathiskumar, D. Gupta and S. Ramakrishna, Osteoblast mineralization with composite nanofibrous substrate for bone tissue regeneration, Cell Biol. Int. 35 (2001) 73-80. 
32. J. M. Martin, J. M. Zenilman and G. S. Lazarus, Molecular microbiology: new dimensions for cutaneous biology and wound healing, J. Invest. Dermatol. 130 (2010) 38-48; DOI: 10.1038/jid. 2009.221.

33. F. Gottrup, B. Jørgesen and T. Karlsmark, News in wound healing and management, Curr. Opin. Support. Palliat. Care 3 (2009) 300-304; DOI: 10.1097/SPC.0bo13e328331d40c.

34. C. H. Lee, H. J. Shin, I. H. Cho, Y. Kang, I. A. Kim, K. D. Park and J. Shin, Nanofiber alignment and direction of mechanical strain affect the ECM production of human ACL fibroblast, Biomaterials 26 (2005) 1261-1270; DOI: 10.1016/j.biomaterials. 2004.04.037.

35. M. Cegnar, S. Baumgartner and J. Kristl, Vlažna oskrba rane s sodobnimi oblogami, Med. Razgl. 46 (2007) 235-247.

36. P. Zahedi, I. Rezaeian, S. O. Ranaei-Siadat, S. H. Jafari and P. Supaphol, A review on wound dressings with an emphasis on electrospunnanofibrous polymeric bandages, Polym. Adv. Technol. 21 (2010) 77-95; DOI: 10.1002/pat.1625.

37. S. Balaji, S. V. Vaikunth, S. A. Lang, A. Q. Sheikh, F. Y. Lim, T. M. Cromleholme and D. A. Narmoneva, Tissue-engineered provisional matrix as a novel approach to enhance diabetic wound healing, Wound Repair Regen. 20 (2012) 15-27; DOI: 10.1111/j.1524-475X.2011.00750.x.

38. R. Uppal, G. N. Ramaswamy, C. Arnold, R. Goodband and Y. Wang, Hyaluronic acid nanofiber wound dressing-production, characterization, and in vivo behaviour, J. Biomed. Mater. Res. B: Appl. Biomaterials 97B (2011) 20-29; DOI: 10.1002/jbm.b.31776.

39. M. Jannesari, J. Varshosaz, M. Morshed and M. Zamani, Composite poly(vinyl alcohol)/poly (vinyl acetate) electrospun nanofibrous mats as novel wound dressing matrix for controlled release of drugs, Int. J. Nanomedicine 6 (2011) 993-1003; DOI: 10.2147/IJN.S17595.

40. Y. Zhang, C. T. Lim, S. Ramakrishna and Z. M. Huang, Recent development of polymer nanofibers for biomedical and biotechnological applications, J. Mater. Sci.: Mater. Med. 15 (2005) 933946; DOI: 10.1007/s10856-005-4428-x.

41. K. Kim, Y. K. Luu, C. Chang, D. Fang, B. S. Hsiao, B. Chu and M. Hadjiargyrou, Incorporation and controlled release of a hydrophilic antibiotic using poly (lactide-co-glycolide)-based electro spun nanofibrous scaffolds, J. Control. Release 98 (2004) 47-56; DOI: 10.1016/j.jconrel.2004.04. 009.

42. M. Zamani, M. Morshed, J. Varshosaz and M. Jannesari, Controlled release of metronidazole benzoate from poly epsilon-caprolactone electrospun nanofibers for periodontal diseases, Eur. J. Pharm. Biopharm. 75 (2010) 179-185; DOI: 10.1016/j.ejpb. 2010.02.002.

43. T. D. J. Heunis and L. M. T. Dicks, Nanofibers offer alternative ways to the treatment of skin infections, J. Biomed. Biotechnol. 2010 (2010) 1-10; DOI: 10.1155/2010/510682.

44. P. Taepaiboon, U. Rungsardthong and P. Supaphol, Drug-loaded electrospun mats of poly(vinyl alcohol) fibers and their release characteristics of our model drugs, Nanotechnology 17 (2006) 2317-2329; DOI: 10.1088/0957-4484/17/9/041.

45. G. Ma, Y. Liu, C. Peng, D. Fang, B. He and J. Nie, Paclitaxel loaded electrospun porous nanofibers as mat potential chemotherapy against prostate cancer, Carbohyd. Polym. 86 (2011) 505512; DOI: 10.1016/j.carbpol.2011.04.082.

46. Y. Yang, T. Xia, W. Zhi, L. Wei, J. Weng, C. Zhang and X. Li, Promotion of skin regeneration in diabetic rats by electrospun core-sheath fibers loaded with basic fibroblast growth factor, Biomaterials 32 (2001) 4243-4254; DOI: 10.1016/j.biomaterials. 2011.02.042.

47. D. Liang, Y. K. Luu, K. Kim, B. S. Hsiao, M. Hadjiargyrou and B. Chu, In vitro non-viral gene delivery with nanofibrous scaffolds, Nucleic Acids Res. 33 (2005) e170; DOI: 10.1093/nar/gni171.

48. J. Y. Chun, H. K. Kang, L. Jeong, Y. O. Kang, J. E. Oh, I. S. Yeo, S. Y. Jung, W. H. Park and B. M. Min, Epidermal cellular response to poly(vinyl alcohol) nanofibers containing silver nanoparticles, Coll. Surf. B: Biointerfaces 78 (2010) 334-342; DOI: 10.1016/ j.colsurfb.2010.03.026. 